\title{
Substrate Diffusion and Consumption in Rectangular Capillary-Tissue Bed
}

\author{
Liang Sun ${ }^{1, *}$
}

\begin{abstract}
A diffusion equation can be used to estimate the oxygen concentration profiles for a highly-regular capillary bed of skeletal muscle. In reality, the capillaries may be arranged more or less randomly in normal tissues. The oxygen concentration, along with its time-wise distribution, may be uneven. Heterogeneity in the tissue bed is much more commonly considered. This article presents a mathematical analysis of the capillary-tissue exchange of substrate in microcirculation in rectangular regions where multiple capillaries are embedded with arbitrary characteristics. A matching technique is used to help solve the associated governing equations.
\end{abstract}

Keywords: diffusion, capillary supply region, microcirculation

\section{Introduction}

A basic mathematical model to estimate the oxygen concentration profiles for a highly regular capillary bed of skeletal muscle was originally introduced by Krogh [3]. Oxygen orientation is modeled by a central capillary surrounded by a circular tissue cylinder. Extensions of the Krogh cylinder model have been made, including polygonal regions of supply, capillary effects, axial dependence, oxygen pressure etc. Homogeneities were usually assumed. However, homogeneities may not be applicable to experiments, and heterogeneity in supply and demand is much more common (Egginton [1] and Middleman [4]).

The method described in the following exploits a matching technique which solves the substrate diffusion within a rectangular tissue domain where multi-capillaries are unevenly or randomly distributed. The ideal case is to have the diffusion equation $\nabla^{2} C=1$ with no flux in or out of the boundary. The pinpoint results benefit from the following: (1) filling of circular regions leaves voids in the tissue area and thus is not suitable to be extended to a greater tissue area; (2) in certain human functional capillaries, such as brain tissues, a square or rectangular region is more suitable (Opitz and Schneider [5]); (3) it is easier to take account of unevenness, heterogeneity and flow variations of multi-capillaries when we study this supply region.

\section{Formulation}

Consider a large rectangular region $\Omega$ of length $\alpha$ and width $\beta$, containing $\mathrm{N}$ capillaries of uneven locations and flux strength. Here flux strength gives the substrate diffusion. Within skeletal muscle any given capillary may be surrounded by distinct fiber types. It follows

\footnotetext{
${ }^{1}$ School of Science and Technology, Georgia Gwinnett College, Lawrenceville, GA

${ }^{*}$ Correspondence: lsun@ggc.edu
} 


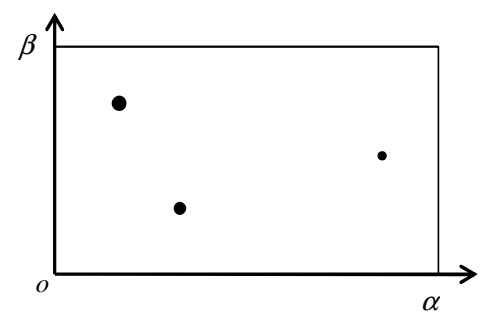

(a)

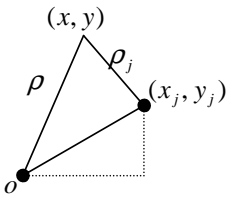

(b)

Figure 1: (a) A general distribution of capillaries in a rectangular region, (b) the coordinate system. Note that $\mathrm{O}$ represents its origin of the Cartesian coordinates.

that diffusion (flux strength) will then depend on local extraction pressures established by differences in consumption (Egginton and Gaffney [2]).

We assume a uniform consumption rate $\kappa$ per volume across $\Omega$. Let $q_{j}$ be the rate of oxygen perfusing into tissue area from the $j$ th capillary source, with radius $\rho_{c}$ centered at $x=x_{j}, \quad y=y_{j}$. Considering the steady state of the oxygen distribution, we have the mass flux balance which gives the following condition on $q_{j}$ :

$$
\sum_{j=1}^{N} q_{j}=\kappa \cdot \alpha \cdot \beta
$$

The governing equation in two dimensions is given by the diffusion equation:

$$
D_{r} \nabla \cdot \nabla C(x, y)=\kappa, \quad C(x, y) \in \Omega,
$$

where $D_{r}$ is the diffusion coefficient of oxygen. On the boundary of the region there is no flux exchange. This is due to the assumption that the target region reaches its steady state:

$$
\begin{aligned}
& \left.\frac{\partial C}{\partial x}\right|_{x \rightarrow 0, \alpha}=0, \\
& \left.\frac{\partial C}{\partial y}\right|_{y \rightarrow 0, \beta}=0 .
\end{aligned}
$$

Let $\left(\rho_{j}, \varphi_{j}\right)$ be local cylindrical coordinates centered at each capillary. Due to consistent flux into the tissue area from the capillary wall, the value of $\kappa$ is zero. The equation for each capillary source is

$$
D_{p}\left(\frac{\partial^{2} c}{\partial \rho_{j}^{2}}+\frac{1}{\rho_{j}} \frac{\partial c}{\partial \rho_{j}}\right)=0 .
$$

The boundary condition for each capillary is

$$
q_{j}=-\left.D_{p} \cdot \rho_{r} \int_{0}^{2 \pi} \frac{\partial c}{\partial \rho_{j}}\right|_{\rho_{j} \rightarrow \rho_{r}} d \varphi_{j}
$$

where $D_{p}$ is the diffusion coefficient in capillary and $\rho_{r}$ is the radius of capillary. Normalizing the flux by $\alpha \beta$ and the diffusion by $D_{p}$, the equations for diffusion in capillaries become 


$$
\left\{\begin{array}{l}
\frac{\partial^{2} c}{\partial \rho_{j}^{2}}+\frac{1}{\rho_{j}} \frac{\partial c}{\partial \rho_{j}}=0 \\
q_{j}=-\left.\frac{\varepsilon}{\pi} \int_{0}^{2 \pi} \frac{\partial c}{\partial \rho_{j}}\right|_{\varepsilon} d \varphi_{j}, \quad \varepsilon=\frac{\rho_{r}}{R} \ll 1 \\
\sum_{j=1}^{N} q_{j}=1 .
\end{array}\right.
$$

Normalizing the concentration in (2) by $\kappa / D_{r}$, the equation for oxygen diffusion in region $\Omega$ becomes

$$
\Delta C(x, y)=1, \quad C(x, y) \in \Omega
$$

with the following boundary conditions:

$$
\left\{\begin{array}{l}
q_{j}=-\left.\frac{\varepsilon}{\pi} \int_{0}^{2 \pi} \frac{\partial c}{\partial \rho_{j}}\right|_{\varepsilon} d \varphi_{j}, \\
\left.\frac{\partial C}{\partial x}\right|_{x \rightarrow 0, \alpha}=0,\left.\quad \frac{\partial C}{\partial y}\right|_{y \rightarrow 0, \beta}=0 .
\end{array}\right.
$$

\section{Matching Technique and Scheme}

Diffusion equations in a rectangular region $\Omega$ with Dirichlet or Neumann boundary conditions have been studied and can be solved by using free Green functions and the method of image. Notice boundary condition (9) is the specific case due to a constant flux out of muticapillaries at different locations, i.e., singular points as $\epsilon \rightarrow 0$ within the region $\Omega$ and it's no longer a simple Neumann problem. Here we use a different strategy for solving Equation (8) to assume general solution in the form

$$
C(x, y)=\frac{x^{2}+y^{2}}{4}-\sum_{j=1}^{N} q_{j} / 2 \cdot \ln \rho_{j}+T(x, y) .
$$

The first term is a particular solution due to uniform consumption. The second term is the combination of fluxes from Equation (7). The last term $T$ is the homogeneous solution which is constructed in order to satisfy the boundary condition on the rectangular region $\Omega$. To satisfy the boundary conditions with no substrate flowing across,we use the process of matching of boundary conditions and apply the series method to construct a suitable function $T(x, y)$.

The boundary conditions are the particular Neumann conditions on a closed rectangular boundary. We separate our solution to satisfy the required boundary conditions on four walls along $x=0, x=\alpha, y=0$ and $y=\beta$. Consider, to begin with, that a series solution has flux zero along $x=\alpha, y=0$ and $y=\beta$ and has an arbitrary sequence of values $\psi_{s}(x)$ (inhomogeneous conditions) along $x=0$. After separation of variables from homogeneous equation associated with Equation (8), the solution of the $x$ equation which has derivative zero at $x=\alpha$ is $\cosh \omega_{\beta}(x-\alpha)$, where $n$ is integer and $\omega_{\beta}$ is the frequency representing $n \pi / \beta$, and the solution of the $y$ equation which has derivative zero at $y=0$ and $y=\beta$ is $\cos \omega_{\beta} y$.

Consequently the most general solution of $\nabla^{2} \psi=0$, in two dimensions, which satisfies the homogeneous Neumann conditions that $\psi$ has derivative zero along $x=\alpha, y=0$ and $y=\beta$ can be represented by the series

$$
\psi=\sum_{n=0}^{\infty} A_{n} \cosh \omega_{\beta}(x-\alpha) \cos \omega_{\beta} y .
$$


The crucial step is to justify the functions in its above series form can satisfy all possible Neumann conditions along $x=0$ and thus can represent all possible solutions satisfying the zero-flux conditions along the three sides. Because we know Neumann conditions along a closed boundary specify a unique solution up to an additive constant, if we find another form of conditions, we can be sure that this new form corresponds to the series and, vice versa, that the series can represent the following new form:

$$
\oint[\psi(z)-F(z)]^{2} d x=0 .
$$

The eigenfunctions $\cos (n \pi y / \beta)$ as a factor in the partial solution (11) given its dependence along the boundary surface depends on a separation constant as well as on position $y$ along the boundary $x=0$. This factor also satisfies the boundary conditions at the ends $y=0$ and $y=\beta$. Here the constants are specially determined to fit the conditions at the chosen boundary sides. The other factor $\cosh \left[\frac{n \pi}{\beta}(x-\alpha)\right]$ is then adjusted to fit the conditions at the other end of the enclosed region $\Omega$ ( $x=0$ in our case), and the complete solution is then the sum of these products for all permissible values of the separation constant.

We have so far only considered the portion $x=0$ of the boundary with boundary values $\psi$. To fit the conditions where $\psi$ is different from zero along other parts of the rectangular boundary we can use obvious modifications of the functions used in series (11). For instance, for fitting conditions along $y=0$ we use the series

$$
\sum_{n=0}^{\infty} D_{n} \cos \omega_{\alpha} x \cosh \omega_{\alpha}(y-\beta)
$$

where $\omega_{\alpha}=n \pi / \alpha$. For $x=\alpha$ and $y=\beta$, we add the individual series to obtain the final solution. Therefore, a corresponding unit function can be derived here to fit the integrand for any boundary values at any point along the rectangular boundary. To solve the Equation (8) and find the unit function in the series, we start with the choice of $T$ for general solution to Equation (8):

$$
T=\psi_{L}+\psi_{R}+\psi_{T}+\psi_{B} .
$$

The four terms can be seen as representations of general solutions against four boundary walls (left, right, top and bottom). Each term involves an unknown coefficient which is to be determined by combination of nonhomogeneous part of equation. And conveniently the four solution terms have the following properties:

$$
\begin{array}{ll}
\left.\frac{\partial T}{\partial x}\right|_{x \rightarrow 0}=\left.\frac{\partial \psi_{L}}{\partial x}\right|_{x \rightarrow 0}, & \left.\frac{\partial T}{\partial x}\right|_{x \rightarrow \alpha}=\left.\frac{\partial \psi_{R}}{\partial x}\right|_{x \rightarrow \alpha}, \\
\left.\frac{\partial T}{\partial y}\right|_{y \rightarrow \beta}=\left.\frac{\partial \psi_{T}}{\partial y}\right|_{y \rightarrow \beta}, & \left.\frac{\partial T}{\partial y}\right|_{y \rightarrow 0}=\left.\frac{\partial \psi_{B}}{\partial y}\right|_{y \rightarrow 0} .
\end{array}
$$

\section{Solution}

The general form of $\mathrm{T}$ is

$$
\begin{aligned}
T= & \sum_{n=0}^{\infty} A_{n} \cosh \omega_{\beta}(x-\alpha) \cos \omega_{\beta} y+\sum_{n=0}^{\infty} B_{n} \cosh \omega_{\beta} x \cos \omega_{\beta} y \\
& +\sum_{n=0}^{\infty} C_{n} \cos \omega_{\alpha} x \cosh \omega_{\alpha} y+\sum_{n=0}^{\infty} D_{n} \cos \omega_{\alpha} x \cosh \omega_{\alpha}(y-\beta) .
\end{aligned}
$$

The relation between $\rho_{j}, x_{j}, y_{j}$ is (Figure $1(\mathrm{~b})$ )

$$
\rho_{j}^{2}=\left(x-x_{j}\right)^{2}+\left(y-y_{j}\right)^{2} .
$$


In order to determine the unknown coefficients $A_{n}, B_{n}, C_{n}$ and $D_{n}$, we look at the boundary conditions given by (3) and (4). The oxygen concentration along each capillary wall depends on the characteristics of the capillary itself; therefore, the radial outward normal at the boundary $\bar{\Omega}$ of (10) gives

$$
\left.\frac{\partial C}{\partial x}\right|_{x \rightarrow 0, \alpha}=\frac{x}{2}-\sum_{j=1}^{N} \frac{q_{j}}{4} \frac{2 x-2 x_{j}}{\left(x-x_{j}\right)^{2}+\left(y-y_{j}\right)^{2}}+\frac{\partial T}{\partial x}=0
$$

and

$$
\left.\frac{\partial C}{\partial y}\right|_{y \rightarrow 0, \beta}=\frac{y}{2}-\sum_{j=1}^{N} \frac{q_{j}}{2} \frac{y-y_{j}}{\left(x-x_{j}\right)^{2}+\left(y-y_{j}\right)^{2}}+\frac{\partial T}{\partial y}=0 .
$$

Equation (16) gives

$$
\begin{aligned}
\frac{\partial T}{\partial x}= & \underbrace{\sum_{n=0}^{\infty} A_{n} \omega_{\beta} \sinh \omega_{\beta}(x-\alpha) \cos \omega_{\beta} y}_{I}+\underbrace{\sum_{n=0}^{\infty} B_{n} \omega_{\beta} \sinh \omega_{\beta} x \cos \omega_{\beta} y}_{I I} \\
& -\underbrace{\sum_{n=0}^{\infty} C_{n} \omega_{\alpha} \sin \omega_{\alpha} x \cosh \omega_{\alpha} y}_{I I I}-\underbrace{\sum_{n=0}^{\infty} D_{n} \omega_{\alpha} \sin \omega_{\alpha} x \cosh \omega_{\alpha}(y-\beta)}_{I V} .
\end{aligned}
$$

As $x \rightarrow 0$ the last three terms tend to approach zero: $I I \rightarrow 0, I I I \rightarrow 0, I V \rightarrow 0$. Therefore

$$
\left.\frac{\partial T}{\partial x}\right|_{x \rightarrow 0}=\sum_{n=0}^{\infty} A_{n} \omega_{\beta} \sinh \omega_{\beta}(-\alpha) \cos \omega_{\beta} y=\left.\frac{\partial \psi_{L}}{\partial x}\right|_{x \rightarrow 0} .
$$

As $x \rightarrow \alpha, I \rightarrow 0, I I I \rightarrow 0, I V \rightarrow 0$

$$
\left.\frac{\partial T}{\partial x}\right|_{x \rightarrow \alpha}=\sum_{n=0}^{\infty} B_{n} \omega_{\beta} \sinh \omega_{\beta} \alpha \cos \omega_{\beta} y=\left.\frac{\partial \psi_{R}}{\partial x}\right|_{x \rightarrow \alpha} .
$$

Equations (21) and (22) give the expression for zero flux flowing towards the boundary $x \rightarrow 0, \alpha$ respectively, and similarly for the vertical boundary lines $y \rightarrow 0, \beta$ :

$$
\begin{aligned}
\frac{\partial T}{\partial y}= & \underbrace{\sum_{n=0}^{\infty}-A_{n} \omega_{\beta}(x-\alpha) \cosh \omega_{\beta}(x-\alpha) \sin \omega_{\beta} y}_{I}-\underbrace{\sum_{n=0}^{\infty} B_{n} \omega_{\beta} \cosh \omega_{\beta} x \sin \omega_{\beta} y}_{I I} \\
& +\underbrace{\sum_{n=0}^{\infty} C_{n} \omega_{\alpha} \cos \omega_{\alpha} x \sinh \omega_{\alpha} y}_{I I I}+\underbrace{\sum_{n=0}^{\infty} D_{n} \omega_{\alpha} \cos \omega_{\alpha} x \sinh \omega_{\alpha}(y-\beta)}_{I V} .
\end{aligned}
$$

As $y \rightarrow 0$, we observe that $I \rightarrow 0, I I \rightarrow 0, I I I \rightarrow 0$. Therefore,

$$
\left.\frac{\partial T}{\partial y}\right|_{y \rightarrow 0}=\sum_{n=0}^{\infty} D_{n} \omega_{\alpha} \sinh \omega_{\alpha}(-\beta) \cos \omega_{\alpha} x=\left.\frac{\partial \psi_{T}}{\partial y}\right|_{y \rightarrow \beta} .
$$

As $y \rightarrow \beta$, we see $I \rightarrow 0, I I \rightarrow 0, I V \rightarrow 0$, and thus,

$$
\left.\frac{\partial T}{\partial y}\right|_{y \rightarrow \beta}=\sum_{n=0}^{\infty} C_{n} \omega_{\alpha} \sinh \omega_{\alpha} \beta \cos \omega_{\alpha} x=\left.\frac{\partial \psi_{B}}{\partial y}\right|_{y \rightarrow 0} .
$$

Equations (21), (22), (24), and (25) show that the form of solution (16) satisfies (14) and (15). Equation (16) is then used to obtain the values for unknown coefficients $A_{n}, B_{n}$, $C_{n}$, and $D_{n}$. 
Using the boundary conditions (18) and (21), as $x \rightarrow 0$, we find

$$
\left.\frac{\partial C}{\partial x}\right|_{x \rightarrow 0}=0+\sum_{j=1}^{N} \frac{q_{j}}{4} \frac{2 x_{j}}{x_{j}^{2}+\left(y-y_{j}\right)^{2}}+\sum_{n=0}^{\infty} A_{n} \frac{n \pi}{\beta} \sinh \left[\frac{n \pi}{\beta}(-\alpha)\right] \cos \frac{n \pi y}{\beta}=0
$$

which implies that

$$
\sum_{n=0}^{\infty} A_{m} \frac{n \pi}{\beta} \sinh \left[\frac{n \pi}{\beta}(-\alpha)\right] \cos \frac{n \pi y}{\beta}=\underbrace{\sum_{j=1}^{N} \frac{q_{j}}{2} \frac{-x_{j}}{x_{j}^{2}+\left(y-y_{j}\right)^{2}}}_{g_{1}(y)} .
$$

Multiplying Equation (27) by $\cos \frac{m \pi}{\beta} y$ and integrating from $-\beta$ to $\beta$ with respect to $y$,

$$
A_{m} \frac{m \pi}{\beta} \sinh \left[\frac{m \pi}{\beta}(-\alpha)\right] \cdot \pi=\int_{-\beta}^{\beta} g_{1}(y) \cdot \cos \frac{m \pi}{\beta} y d y
$$

where $g_{1}(y)=\sum_{j=1}^{N} \frac{q_{j}}{2} \frac{-x_{j}}{x_{j}^{2}+\left(y-y_{j}\right)^{2}}$. Let $Y=\left(y-y_{j}\right)^{2}$. Equation (28) yields

$$
\begin{aligned}
A_{m} \frac{m \pi}{\beta} & \sinh \left[\frac{m \pi}{\beta}(-\alpha)\right] \cdot \pi \\
& =\sum_{j=1}^{N} \frac{q_{j}}{2} \int_{-\beta}^{\beta} \frac{-x_{j}}{x_{j}^{2}+(Y)^{2}}\left(\cos \frac{m \pi}{\beta} Y \cos \frac{m \pi}{\beta} y_{j}-\sin \frac{m \pi}{\beta} Y \sin \frac{m \pi}{\beta} y_{j}\right) d Y \\
& =\sum_{j=1}^{N} \frac{q_{j}}{2} \int_{-\beta}^{\beta} \frac{-x_{j}}{x_{j}^{2}+(Y)^{2}} \cos \frac{m \pi}{\beta} Y \cos \frac{m \pi}{\beta} y_{j} d Y \\
& =\sum_{j=1}^{N} \frac{q_{j}}{2}\left(-x_{j}\right) 2 \cos \frac{m \pi}{\beta} y_{j} \int_{0}^{\beta} \frac{1}{x_{j}^{2}+(Y)^{2}} \cos \frac{m \pi}{\beta} Y d Y
\end{aligned}
$$

Thus

$$
A_{m}=\frac{1}{m \pi \sinh \frac{m \pi}{\beta}(-\alpha)} \sum_{j=1}^{N} q_{j}\left(-x_{j}\right) \cos \frac{m \pi}{\beta} y_{j} \int_{0}^{\pi} \frac{1}{\frac{\pi^{2}}{\beta^{2}} x_{j}^{2}+(y)^{2}} \cdot \cos m y d y
$$

Here $y=\frac{m \pi}{\beta} Y$, and again $\left(x_{j}, y_{j}\right)$ gives the location of the $j$ th source. Similarly, we achieve the values for the unknown coefficients $B_{m}, C_{m}$, and $D_{m}$

$$
\begin{aligned}
B_{m} & =\frac{1}{m \pi \sinh \frac{m \pi}{\beta}(\alpha)} \sum_{j=1}^{N} q_{j}\left(\alpha-x_{j}\right) \cos \frac{m \pi}{\beta} y_{j} \int_{0}^{\pi} \frac{1}{\frac{\pi^{2}}{\beta^{2}}\left(\alpha-x_{j}\right)^{2}+(y)^{2}} \cdot \cos m y d y \\
C_{m} & =\frac{1}{m \pi \sinh \frac{m \pi}{\alpha}(\beta)} \sum_{j=1}^{N} q_{j}\left(\beta-y_{j}\right) \cos \frac{m \pi}{\alpha} x_{j} \int_{0}^{\pi} \frac{1}{(x)^{2}+\frac{\pi^{2}}{\alpha^{2}} y_{j}^{2}} \cdot \cos m x d x \\
D_{m} & =\frac{1}{m \pi \sinh \frac{m \pi}{\alpha}(-\beta)} \sum_{j=1}^{N} q_{j}\left(-y_{j}\right) \cos \frac{m \pi}{\alpha} x_{j} \int_{0}^{\pi} \frac{1}{(x)^{2}+\frac{\pi^{2}}{\alpha^{2}}\left(\beta-y_{j}\right)^{2}} \cdot \cos m x d x
\end{aligned}
$$

\section{$5 \quad$ Examples and Discussion}

Oxygen delivery to tissue has been studied in various ways. Popel [6], [7] used evenly distributed domains for multi-capillaries with heterogeneous flow. Schmidt-Nielsen and Pennycuik [8] discovered a triangular regional feature for white skeletal muscles with capillaryto-fiber ratio approximately equal to one. Opitz and Schneider [5] used a square region of 
supply for brain capillaries. These authors used polygonal region for capillary supply rather than a disk and estimated minimum tissue pressure within the supply region. But their target tissue contains only one single capillary and they assumed a uniform distribution of capillary locations across the whole tissue region. A circular region was studied by Wang [9] and analytical solutions were given. However, tiled circular regions will leave voids in tissue area and therefore can not represent the entire tissue region. Rectangular regions can be tiled without voids into sub tissue regions. We have applied a matching technique to achieve complete solutions for oxygen concentration through a rectangular region. Some examples will be illustrated in the following. In Example III, nine capillaries with random characteristics will be used to illustrate concentration level from using its analytical solution. The number of capillaries can always be increased in experiments. Note that complexity of the analytical solutions in series form requires large computing time if the number of capillaries becomes large (e.g., greater than 50). Future work will focus on refining the solutions in series form and reducing the amount of time for computing large group of capillaries.

\subsection{A single capillary in a diffusion-consumption rectangular region with $\alpha=1$ and $\beta=1$}

Consider a single capillary in the center of the region with flux strength $q=1$. The results for coefficients are displayed in Table 1 to show the convergence of the series to the entire rectangular region. We also show the plot of oxygen concentration $C(x, y)$ in Figure 2. We have truncated to only the first 40 terms in each set of coefficients. It reaches our accuracy demand.

\begin{tabular}{c|ccccc|} 
& $n=1$ & $n=2$ & $n=10$ & $n=20$ & $n=30$ \\
\hline$A_{n}$ & -0.1019 & -0.1006 & $1.066 \times 10^{-5}$ & $-1.796 \times 10^{-6}$ & $-3.001 \times 10^{-9}$ \\
$B_{n}$ & -0.1024 & -0.1023 & $1.080 \times 10^{-5}$ & $-1.659 \times 10^{-6}$ & $-3.025 \times 10^{-8}$ \\
$C_{n}$ & -0.1024 & -0.1023 & $1.080 \times 10^{-5}$ & $-1.659 \times 10^{-6}$ & $-3.025 \times 10^{-8}$ \\
$D_{n}$ & -0.1019 & -0.1006 & $1.066 \times 10^{-5}$ & $-1.796 \times 10^{-6}$ & $-3.001 \times 10^{-9}$ \\
\hline
\end{tabular}

Table 1: Coefficients of Example I for a rectangular domain up to $n=30$ with $\alpha=1$ and $\beta=1$

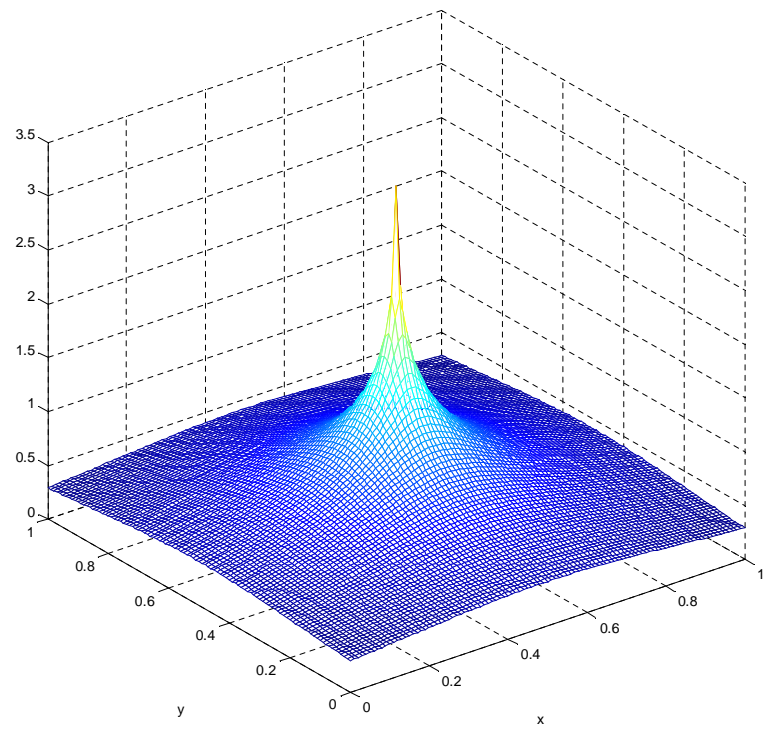

Figure 2: Oxygen concentration in its steady state with one single capillary inside a sufficiently oxygenated rectangular domain, $\alpha=1$ and $\beta=1$ in Example I. 


\subsection{Three random single capillaries in a well-supplied diffusion- consumption rectangular region with $\alpha=1$ and $\beta=1$}

Consider three capillaries of uneven strength in a rectangular region. Locations and flux strengths of the capillaries shown in Table 2 are randomly generated with the sum of flux equal to 1 . The results for coefficients are shown in Table 3 to show the convergence of the solution to the entire rectangular region. We also show the plot of oxygen concentration $C(x, y)$ in Figure 3. We have truncated to only the first 40 terms in each set of coefficients since it reaches our accuracy goal.

\begin{tabular}{c|ccc|}
$k$ & $x_{k}$ & $y_{k}$ & $q_{k}$ \\
\hline 1 & 0.721 & 0.637 & 0.176 \\
2 & 0.812 & 0.243 & 0.412 \\
3 & 0.175 & 0.320 & 0.412 \\
\hline
\end{tabular}

Table 2: Locations and flux strengths of three capillaries in Example II

\begin{tabular}{c|ccccc|} 
& $n=1$ & $n=2$ & $n=10$ & $n=20$ & $n=30$ \\
\hline$A_{n}$ & 0.0035 & -0.0003 & $-1.160 \times 10^{-6}$ & $-1.310 \times 10^{-8}$ & $1.227 \times 10^{-10}$ \\
$B_{n}$ & 0.0037 & -0.0006 & $-5.216 \times 10^{-7}$ & $-1.804 \times 10^{-8}$ & $-3.078 \times 10^{-10}$ \\
$C_{n}$ & -0.6731 & -0.0012 & $8.478 \times 10^{-5}$ & $-5.249 \times 10^{-8}$ & $4.606 \times 10^{-9}$ \\
$D_{n}$ & -0.0011 & 0.0003 & $1.153 \times 10^{-7}$ & $-7.940 \times 10^{-9}$ & $1.049 \times 10^{-10}$ \\
\hline
\end{tabular}

Table 3: Coefficients of Example II for a rectangular domain up to $n=30$ with $\alpha=1$ and $\beta=1$

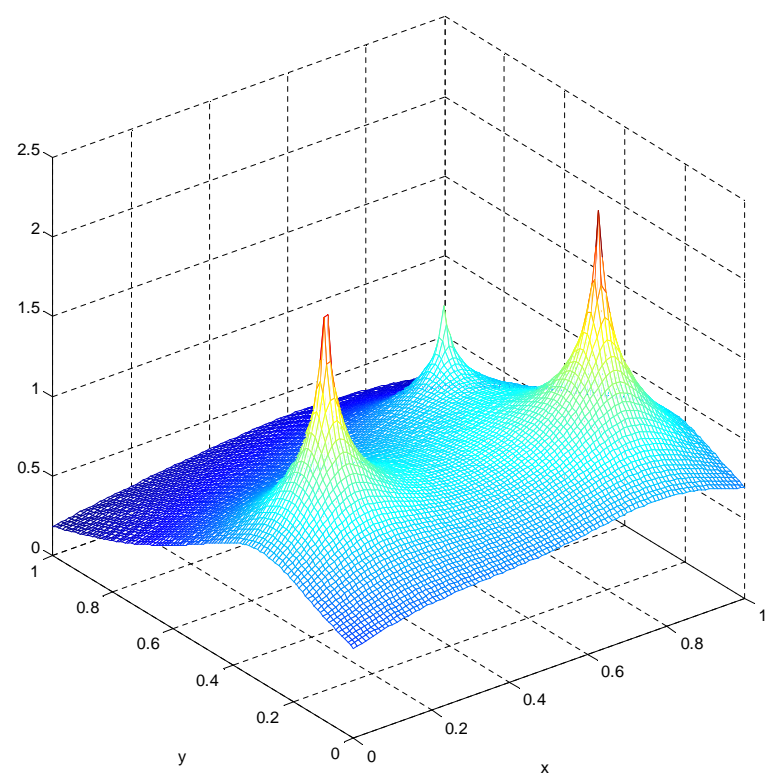

Figure 3: Oxygen concentration in its steady state with three capillaries inside a sufficiently oxygenated rectangular domain, $\alpha=1$ and $\beta=1$ in Example II. 


\subsection{Nine random single capillaries in a well-sufficed diffusion-con- sumption rectangular region with $\alpha=1$ and $\beta=1$ and plot of contour}

Consider nine capillaries of uneven strength in a rectangular region. Locations and flux strengths of the capillaries are randomly generated, shown in Table 4, with the sum of flux equal to 1 . The results for coefficients are given in Table 5 and 6 to show the convergence of the solution in the entire rectangular region. We also show the plot of oxygen concentration level curve in Figure 4. We have truncated to only the first 40 terms in each set of coefficients.

\begin{tabular}{c|ccc|}
$k$ & $x_{k}$ & $y_{k}$ & $q_{k}$ \\
\hline 1 & 0.501 & 0.498 & 0.044 \\
2 & 0.721 & 0.235 & 0.089 \\
3 & 0.242 & 0.754 & 0.133 \\
4 & 0.091 & 0.046 & 0.133 \\
5 & 0.633 & 0.923 & 0.089 \\
6 & 0.867 & 0.354 & 0.023 \\
7 & 0.425 & 0.301 & 0.133 \\
8 & 0.664 & 0.316 & 0.178 \\
9 & 0.723 & 0.833 & 0.178 \\
\hline
\end{tabular}

Table 4: Locations and flux strengths of nine capillaries in Example III

\begin{tabular}{c|ccccc|} 
& $n=1$ & $n=2$ & $n=10$ & $n=20$ & $n=30$ \\
\hline$A_{n}$ & -0.2884 & 0.0065 & $1.534 \times 10^{-4}$ & $2.615 \times 10^{-6}$ & $8.110 \times 10^{-8}$ \\
$B_{n}$ & -0.4366 & 0.0060 & $-5.671 \times 10^{-4}$ & $-6.174 \times 10^{-6}$ & $1.099 \times 10^{-7}$ \\
$C_{n}$ & -0.2591 & -0.0047 & $1.790 \times 10^{-4}$ & $-2.513 \times 10^{-6}$ & $-6.557 \times 10^{-8}$ \\
$D_{n}$ & 0.2254 & 0.0049 & $1.906 \times 10^{-4}$ & $2.441 \times 10^{-6}$ & $3.121 \times 10^{-8}$ \\
\hline
\end{tabular}

Table 5: Coefficients of Example III for a rectangular domain up to $n=30$ with $\alpha=1$ and $\beta=1$.

\begin{tabular}{c|ccc} 
& $n=40$ & $n=50$ & $n=60$ \\
\hline$A_{n}$ & $1.690 \times 10^{-12}$ & $3.871 \times 10^{-14}$ & $8.775 \times 10^{-16}$ \\
$B_{n}$ & $8.553 \times 10^{-14}$ & $3.693 \times 10^{-15}$ & $1.263 \times 10^{-16}$ \\
$C_{n}$ & $2.184 \times 10^{-12}$ & $-8.853 \times 10^{-16}$ & $-1.608 \times 10^{-15}$ \\
$D_{n}$ & $-8.142 \times 10^{-13}$ & $-8.648 \times 10^{-14}$ & $4.239 \times 10^{-15}$ \\
\hline
\end{tabular}

Table 6: Coefficients of Example III for a rectangular domain up from $n=40$ to $n=60$ with $\alpha=1$ and $\beta=1$. 


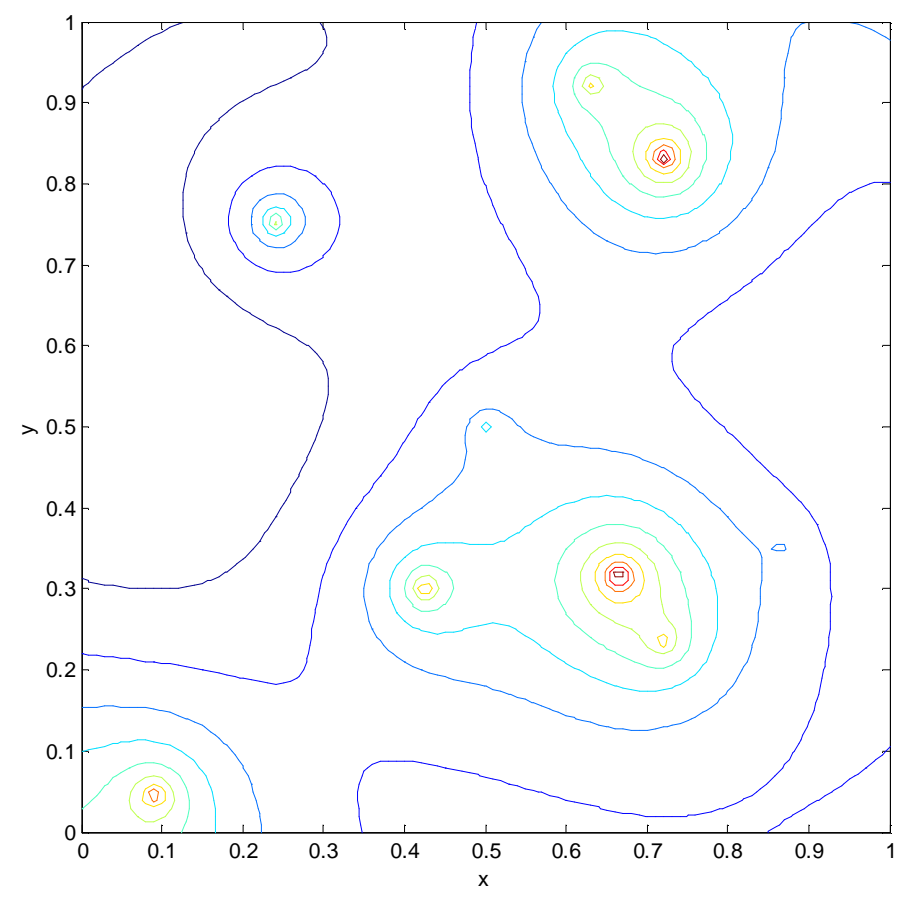

Figure 4: Oxygen concentration level curve in a sufficiently oxygenated rectangular domain with nine capillaries, steady state, $\alpha=1$ and $\beta=1$ in Example III.

\section{References}

[1] S. Egginton, Anatomical adaptations for peripheral oxygen transport at high and low temperatures. S Afr J Zool 33, 119-128, 1998.

[2] S. Egginton and E. Gaffney, Experimental Physiology — Review Article: Tissue capillary supply - it's quality not quantity that counts! expphysiol 95:971-979, 2010.

[3] A. Krogh, The number and distribution of capillaries in muscles with calculations of the oxygen pressure head necessary for supplying the tissue, J.Physiol., 52, 1919.

[4] S. Middleman, Transport phenomena in the cardiovascular system, Wiley-Interscience, New York, 1972.

[5] E. Opitz and M. Schneider, The oxygen supply of the brain and the mechanism of deficiency effects,Ergeb. Physiol. Biol. Chem. Exptl. Pharmakol., 46: 126-260, 1950.

[6] A. S. Popel, Oxygen diffusive shunts under conditions of heterogeneous oxygen delivery, J. Theor. Biol., 96 533, 1982.

[7] A. S. Popel, Analysis of capillary-tissue diffusion in multicapillary systems. Math. Biosci., 39:187-211, 1978.

[8] K. Schmidt-Nielsen, P. Pennycuik, Capillary density in mammals in relation to body size and oxygen consumption Amer. J. Physiol., 200:746-760, 1961.

[9] C. Y. Wang, J. B. Bassingthwaighte, Capillary supply regions, Math. Biosci., 173 103, 2001. 